\title{
Estudio comparativo de precisión diagnóstica preoperatoria y la confirmación laparoscópica de hernia hiatal
}

\author{
Italo Braghetto M. ${ }^{1}$, Nicole Cuneo B. ${ }^{1}$, Marcelo Zamorano D. ${ }^{1}$, \\ Jorge Rojas C. ${ }^{1}$, Amy Piñeres A. ${ }^{1}$ y Manuel Figueroa G. ${ }^{1}$
}

\section{Comparative study of preoperative diagnostic precision and laparoscopic confirmation of hiatal hernia}

\begin{abstract}
Introduction: Being type I hiatal hernia $(\mathrm{HH})$ the most frequent, is difficult to define objectively and therefore, the main focus of controversy is the diagnosis. The aim of this paper is to report the results regarding the diagnostic accuracy of the preoperative study and to confirm it with the laparoscopic diagnosis of hiatal hernia. Materials and Method: This descriptive and prospective study includes patients with typical symptoms of gastroesophageal reflux disease who underwent esophageal-gastro-duodenoscopy, manometry and radiological study of esophagus with barium swallow. Only patients in whom endoscopy reveals the existence of $\mathrm{HH}$ by sliding $<5 \mathrm{~cm}$ are included. These patients underwent surgical treatment confirming or not the existence of $\mathrm{HH}$ at the time of laparoscopic exploration. Results: The positive predictive value and sensibility for manometry was $51.2 \%$ and $70 \%$, for radiology $91.7 \%$ and $80.5 \%$, and for endoscopy $85.3 \%$ and $70.7 \%$ respectively. Conclusion: For the reliable diagnosis of $\mathrm{HH}$ before treatment, the three mentioned investigations must be mandatory before the surgery.

Key words: hiatal hernia; manometry; endoscopy; laparoscopy; diagnosis.
\end{abstract}

\section{Resumen}

Introducción: La hernia hiatal $(\mathrm{HH})$ de tipo I por deslizamiento es el tipo más frecuente, siendo difícil de definir objetivamente, por lo que el principal foco de controversia es su diagnóstico. El objetivo del presente trabajo es reportar los resultados respecto de la precisión diagnóstica del estudio preoperatorio y confirmarlo con el diagnóstico laparoscópico de este tipo de HH. Materiales y Método: Estudio prospectivo descriptivo de serie que incluyen pacientes con síntomas típicos de enfermedad por reflujo gastroesofágico, los cuales se sometieron a estudio con esófago-gastro-duodenoscopía, estudio manométrico y radiológico de esófago, estómago y duodeno con bario. Se incluyen sólo los pacientes en los cuales la endoscopía revela la existencia de $\mathrm{HH}$ por deslizamiento $<5 \mathrm{~cm}$. Estos pacientes se sometieron a tratamiento quirúrgico confirmándose o no la existencia de $\mathrm{HH}$ al momento de la exploración laparoscópica. Resultados: El valor predictivo positivo y sensibilidad para manometría fue de un 51,2\% y 70\%, para la radiología 91,7\% y $80,5 \%$ y para endoscopia $80,3 \%$ y $70,7 \%$ respectivamente. Conclusión: Para el diagnóstico confiable de $\mathrm{HH}$ antes del tratamiento, las tres investigaciones mencionadas deben ser obligatorias antes de la cirugía. Palabras clave: hernia hiatal; manometría; endoscopía; laparoscopía; diagnóstico.

\section{Introducción}

La hernia hiatal $(\mathrm{HH})$ de tipo I por deslizamiento, si bien es el tipo más frecuente, es la más difícil de definir objetivamente y principal foco de controversia en su diagnóstico. La HH tipo I por deslizamiento se diagnostica comúnmente con endoscopía digestiva alta, radiología de esófagoestómago-duodeno con trago de bario y con menos frecuencia, con manometría esofágica. Hernias hiatales por deslizamiento mayores de $5 \mathrm{~cm}$, no representan mayor problema, pero en menores de $5 \mathrm{~cm}$ el diagnóstico clínico es un problema ya que existe mucha variabilidad y confusión conceptual.
'Departamento de Cirugía, Hospital Clínico Universidad de Chile. Santiago, Chile.

Recibido el 4 de julio de 2019 y aceptado para publicación el 28 de octubre de 2019.

Correspondencia a:

Italo Braghetto M. ibragheto@hcuch.cl 
Para algunos la radiología baritada es el método que ofrece mayor precisión en el diagnóstico, puede determinar su tamaño y es el método que mejor puede detectar una imagen de HH y la unión gastroesofágica (UGE). Entre los endoscopistas existe gran variabilidad en la interpretación de los hallazgos en cuanto a la determinación de la UGE, límite del cambio de epitelios y la visualización de los pliegues gástricos. Mayor aún es la controversia si el paciente presenta signos endoscópicos de esófago de Barrett. Mediante la manometría el hallazgo de un esfínter desplazado a tórax es sugerente de $\mathrm{HH}$, sin embargo, este método tiene problemas ya sea con manometría convencional como manometría de alta resolución debido a la movilidad de la UGE. En cuanto a la precisión diagnóstica existen diferentes opiniones respecto de los métodos empleados, y el diagnóstico de certeza se confirma durante la exploración quirúrgica laparoscópica al observarse un saco herniario que se invagina hacia el mediastino ${ }^{1,2}$.

El objetivo del presente trabajo es reportar la precisión diagnóstica del estudio preoperatorio y confirmarlo con el diagnóstico intraoperatorio de HH tipo I por deslizamiento.

\section{Materiales y Método}

Estudio prospectivo descriptivo de serie que incluyen pacientes con síntomas típicos de enfermedad por reflujo gastroesofágico estudiados con esófago-gastro-duodenoscopía, manometría, (35 con manometría clásica y 15 con manometría de alta resolución) y radiología de esófago, estómago y duodeno con bario. Se incluyen sólo los pacientes en los cuales la endoscopía revela la existencia de $\mathrm{HH}$ por deslizamiento menores de $5 \mathrm{~cm}$. Estos pacientes se sometieron a tratamiento quirúrgico confirmándose o no la existencia de $\mathrm{HH}$ al momento de la exploración laparoscópica.

\section{Criterio de exclusión}

Se excluyen las grandes $\mathrm{HH}$ de mayor tamaño $(>5 \mathrm{~cm})$ sean de cualquier tipo por no existir dudas en el diagnóstico preoperatorio.

\section{Definición de hernia hiatal}

Manométricamente, la $\mathrm{HH}$ se define como la separación de la unión gastroesofágica (UGE o cardias) $>2 \mathrm{~cm}$ del diafragma crural, determinado por la posición del punto de inversión de las presiones que es el límite básico para el diagnóstico de hernia por deslizamiento. Otro parámetro a observar en manometría convencional es la ausencia de esófago abdominal ${ }^{1,2}$.

Endoscópicamente se diagnostica cuando la separación entre la unión escamo-columnar y la impresión diafragmática es mayor de $2 \mathrm{~cm}$, y existen pliegues gástricos que emergen desde distal o bien la observación de un cardias dilatado tipo III de la clasificación de Hill-7 (Figura 1).

Radiológicamente se define cuando la UGE o cardias se ubica al menos $2 \mathrm{~cm}$ por encima del hiato diafragmático y pliegues gástricos que ascienden por el hiato esofágico ${ }^{2-7}$ (Figura 1).

Al inicio de la exploración laparoscópica es el momento en que se confirma objetivamente la existencia de una hernia hiatal tipo I deslizante mediante la visualización de un saco herniario a través del hiato diafragmático dilatado, independiente de su tamaño (Figura 1). Las otras hernias tipo II, III o IV de mayor tamaño no presentan confusión diagnóstica $^{2,8,9}$.

\section{Análisis estadístico}

Para el análisis estadístico, se evaluó la población descriptivamente. Los estudios preoperatorios se compararon en una tabla de contingencia con el gold-standard que se consideró la laparoscopía $\left(\chi^{2}\right.$, $\mathrm{p}$ menor 0,05$)$. También se analizaron los estudios preoperatorios en comparación con la radiografía esófago-estómago-duodeno que se considera el goldstandard para el diagnóstico preoperatorio de esta patología $\left(\chi^{2}, \mathrm{p}\right.$ menor 0,05$)$. Todo el estudio estadístico se realizó mediante el programa SPSS ${ }^{\circledR}$ v. 21 .

\section{Resultados}

Este estudio prospectivo incluye 50 pacientes (33 mujeres - 17 hombres), sometidos a tratamiento quirúrgico por vía laparoscópica por reflujo gastroesofágico y sospecha de hernia hiatal. Se confirmó la existencia hernia hiatal laparoscópica en 41 pacientes. En la Tabla 1 se muestran los resultados del estudio manométrico y su confirmación al momento de la laparoscopía. En los 41 pacientes con hernia hiatal laparoscópica, en solo 21 de ellos, la manometría reveló la existencia de una hernia hiatal. El valor predictivo positivo (VPP) fue de un $51,2 \%$, la sensibilidad (S) de un 70\%, especificidad y valor predictivo negativo (VPN) ambos $0 \%$. En cambio, para el estudio radiológico preoperatorio el valor predictivo positivo fue de un $80,5 \%$ y la sensibilidad de un $91,7 \%$ y una especificidad mayor (p value 0,004) (Tabla 2). Resultados similares se encontraron al analizar el diagnóstico endoscópico 
y los hallazgos laparoscópicos, con una sensibilidad de 85,3\%, especificidad (E) de $25 \%$ y un VPP de 70,7\% (Tabla 3). Al analizar los resultados entre el diagnóstico radiológico y manométrico el valor predictivo positivo es de solo un 60\% (Tabla 4). Finalmente, en la Tabla 5 se analiza el diagnóstico endoscópico versus el diagnóstico manométrico y los resultados son bastante similares ( $\mathrm{p}$ value 0,70). En la Figura 1 se muestra un resumen final del cuadro conceptual del trabajo y los resultados obtenidos con los diferentes métodos usados para el diagnóstico de una hernia hiatal tipo I por deslizamiento.

Tabla 1. Estudio manométrico preoperatorio y confirmación laparoscópica de hernia hiatal tipo I $(\mathbf{n}=\mathbf{5 0})$

\begin{tabular}{|lrcc|}
\hline $\begin{array}{l}\text { Laparoscopía } \\
\text { hernia hiatal }\end{array}$ & n & $\begin{array}{c}\text { Hernia hiatal manométrica } \\
\text { Presente }\end{array}$ & $\begin{array}{c}\text { Ausente } \\
\text { Presente }\end{array}$ \\
Ausente & 91 & 21 & 20 \\
Total & 50 & 9 & 0 \\
\hline
\end{tabular}

p value no calculable. S: 70\%. E: 0\%. VPP: 51,2\%. VPN: $0 \%$.

Tabla 2. Estudio radiológico preoperatorio y confirmación laparoscópica de hernia hiatal tipo I $(n=50)$

\begin{tabular}{|c|c|c|c|}
\hline \multirow{2}{*}{$\begin{array}{l}\text { Laparoscopía } \\
\text { hernia hiatal }\end{array}$} & \multirow[b]{2}{*}{$\mathbf{n}$} & \multicolumn{2}{|c|}{ Hernia hiatal radiológica } \\
\hline & & Presente & Ausente \\
\hline Presente & 41 & 33 & 8 \\
\hline Ausente & 9 & 3 & 6 \\
\hline Total & 50 & 36 & 14 \\
\hline
\end{tabular}

p value 0,004 . S: $91,7 \%$. E: 42,9\%. VPP: $80,5 \%$. VPN: $66,7 \%$.
Tabla 3. Estudio endoscópico preoperatorio y confirmación laparoscópica de hernia hiatal tipo I $(n=50)$

\begin{tabular}{|lccc|}
\hline $\begin{array}{l}\text { Laparoscopía } \\
\text { hernia hiatal }\end{array}$ & n & \multicolumn{2}{c|}{ Hernia hiatal endoscópica } \\
Presente & Ausente \\
Presente & 41 & 29 & 12 \\
Ausente & 9 & 5 & 4 \\
Total & 50 & 34 & 16 \\
\hline
\end{tabular}

p value 0,38 . S: $85,3 \%$. E: $25 \%$. VPP: 70,7\%. VPN: 44,4\%.

Tabla 4. Estudio radiológico preoperatorio y manometría preoperatoria en hernia hiatal tipo I $(n=50)$

\begin{tabular}{|lccc|}
\hline $\begin{array}{l}\text { Radiología } \\
\text { hernia hiatal }\end{array}$ & n & $\begin{array}{c}\text { Hernia hiatal manométrica } \\
\text { Presente }\end{array}$ & $\begin{array}{c}\text { Ausente } \\
\text { Presente }\end{array}$ \\
Ausente & 14 & 21 & 15 \\
Total & 50 & 9 & 5 \\
\hline
\end{tabular}

p value 0,70. S: 70\%. E: 25\%. VPP: 58,3\%. VPN: 35,7\%.

Tabla 5. Estudio endoscópico preoperatorio y manometría preoperatoria en hernia hiatal tipo $I(n=50)$

\begin{tabular}{|c|c|c|c|}
\hline \multirow{2}{*}{$\begin{array}{l}\text { Endoscopía } \\
\text { hernia hiatal }\end{array}$} & \multirow[b]{2}{*}{$\mathbf{n}$} & \multicolumn{2}{|c|}{ Hernia hiatal manométrica } \\
\hline & & Presente & Ausente \\
\hline Presente & 34 & 21 & 13 \\
\hline Ausente & 16 & 9 & 7 \\
\hline Total & 50 & 30 & 20 \\
\hline
\end{tabular}

p value $0,71$. S: $70 \%$. E: $35 \%$. VPP: $61,8 \%$. VPN: $43,8 \%$.

\section{Estudio comparativo de precisión diagnóstica de la radiología, endoscopía y manometría con la confirmación diagnóstica de hernia hiatal por deslizamiento durante la exploración laparoscópica.}

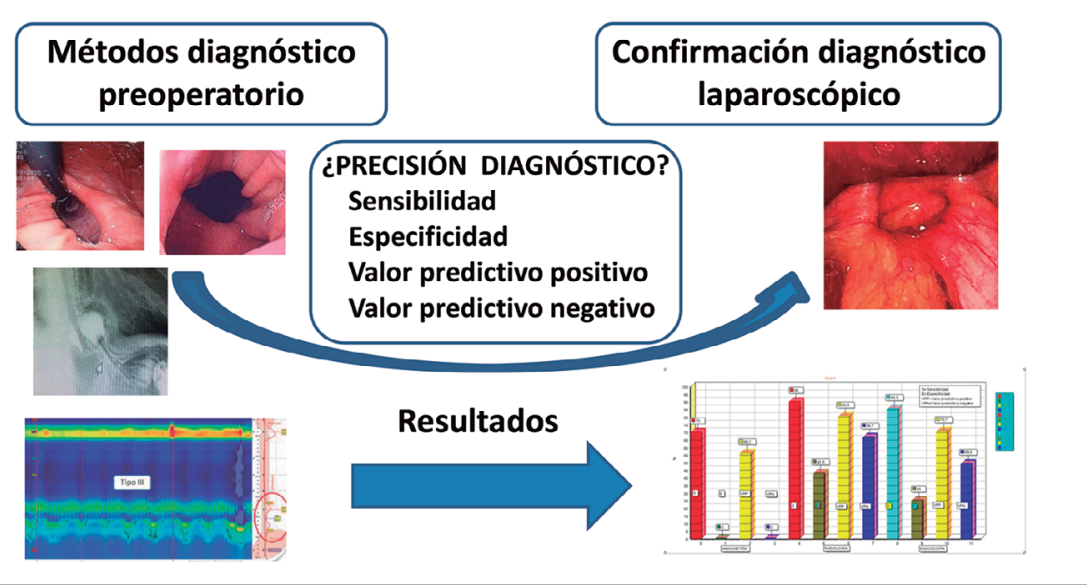

Figura 1. Resumen conceptual y resultados comparativos de los diferentes métodos en el diagnóstico de hernia hiatal por deslizamiento tipo I. 


\section{Discusión}

El esfínter gastroesofágico (EGE) es una estructura móvil y la deglución, la distensión esofágica o la instrumentación esofágica se asocian con desplazamiento de la unión gastroesofágica que podrían inducir a error. Las hernias de hiato de tipo I son las más frecuentes, pero son los más difíciles de definir objetivamente. Los tipos II, III y IV son menos frecuentes y no hay mayor discusión en su diagnóstico?.

La demostración radiológica de pliegues gástricos que atraviesan el diafragma se usa como criterio definitorio. Para el diagnóstico de una hernia de hiato deslizante se requiere una separación de más de $2 \mathrm{~cm}$ entre la EGE-UGE y el hiato diafragmático, menores magnitudes de separación se consideran fisiológicas, lo cual es bastante variable. Lo más importante en el examen radiológico con bario es la observación de la exacta localización de la impresión del hiato diafragmático y la presencia de pliegues gástricos ascendentes, un 15\% de los individuos presentan un anillo de Schatzky, pero la identificación de hernias de tipo I de menos de $3 \mathrm{~cm}$ de tamaño no es confiable ${ }^{8,9}$.

Hay poca uniformidad o rigor en la evaluación endoscópica de la hernia de hiato tipo I por deslizamiento. La exactitud y la reproducibilidad tienen las mismas limitaciones que las mediciones realizadas durante la radiografía con bario. La existencia de un esófago de Barrett dificulta la localización del EGE y UGE, un exceso de insuflación del estómago podría exagerar el tamaño aparente de la hernia. Otro aspecto para la clasificación endoscópica de la $\mathrm{HH}$ por deslizamiento es evaluar la apariencia de la UGE con retrovisión endoscópica, pero existe gran variabilidad en la apreciación de este aspecto $^{5,6,10}$. Hay pocos estudios de la sensibilidad o reproducibilidad de la clasificación endoscópica, los datos recientes sugieren que la endoscopía puede proporcionar información valiosa con respecto del diámetro cardial y existencia de pliegues que se invaginan hacia proximal durante la retrovisión y a menos que se cumplan los criterios diagnósticos, la identificación de hernias de tipo I de menos de $3 \mathrm{~cm}$ de tamaño con endoscopía es poco confiable 9 .

El estudio manométrico presenta aspectos que inciden en la variabilidad de los resultados tales como la magnitud de la presión del EGE, la magnitud del aumento de presión asociado con la contracción de la crura diafragmática, la magnitud de la diferencia presión intragástrica e intraesofágica y lo más importante la separación axial entre el EGE-UGE y el hiato diafragmático. La correlación de los hitos anatómicos y la posición del EGE, representan solo un instante en el tiempo y puede existir mucha variación dado el desplazamiento fisiológico del EGE durante la deglución y los movimientos respiratorios, aunque la manometría de alta resolución es más precisa ${ }^{11,12}$. Se ha reportado que falsos positivos fueron significativamente menos (mayor especificidad) con la manometría de alta resolución en comparación con la endoscopia $(4,88 \%$ frente a $31,71 \%, p=0,01)$. No se han reportado diferencias significativas en falsos negativos (sensibilidad) entre las dos modalidades de diagnóstico $(47,62 \%$ vs $45,24 \% \%^{9,11,12}$. Debido a la baja sensibilidad, tanto la manometría de alta resolución como la endoscopia, no son confiables para descartar una hernia hiatal ${ }^{12}$.

Un estudio de Agrawal ${ }^{13}$, en pacientes con síntomas de reflujo para evaluar la prevalencia de $\mathrm{HH}$, la endoscopía hizo el diagnóstico en un 33\% y manometría en $7 \%$. La sensibilidad de la manometría fue muy baja (20\%), buena especificidad (99\%), valor predictivo positivo $91 \%$ y valor predictivo negativo $66 \%$. Concluye que la manometría infrecuentemente identifica la presencia de HH. Resultados similares fueron reportados también por Klaus ${ }^{14}$.

Nuestros resultados son bastante similares a lo reportado en la literatura ${ }^{14-16}$. Weitzendorfer ${ }^{15}$, encontró que la manometría de alta resolución hizo el diagnóstico de $\mathrm{HH}$ en el 31\%, con tamaño promedio de $3 \mathrm{~cm}$, con endoscopía el diagnóstico fue de $48 \%$ y con radiología el porcentaje de diagnóstico fue de $77 \%$. Otros estudios refieren que la radiología con bario es el método más sensitivo, pero con la manometría de alta resolución se puede detectar muy bien las hernias hiatales y precisar su tamaño, sin embargo, los resultados comparado a la radiología no siempre coinciden ${ }^{1,15,16}$.

\section{Conclusión}

El diagnóstico de hernia hiatal por deslizamiento tipo I pequeña es un desafío diagnóstico a raíz de la variabilidad de los métodos actuales, En definitiva, para el diagnóstico confiable de $\mathrm{HH}$ antes del tratamiento, las tres investigaciones mencionadas deben ser obligatorias antes de la cirugía ${ }^{12,13}$.

\section{Responsabilidades éticas}

Este artículo no contiene estudios experimentales en humanos ni animales.

Este estudio ha sido efectuado de acuerdo a las normas éticas de nuestro hospital y de acuerdo a la declaración de Helsinky.

Conflictos de interés: Los autores de ese trabajo declaran no tener conflictos de interés. 


\section{Bibliografía}

1. Khajanchee YS, Cassera MA, Swanström LL, Dunst CM. Diagnosis of Type-I hiatal hernia: a comparison of highresolution manometry and endoscopy. Dis Esophagus. 2013;26:1-6.

2. Ortega JA, Pérez L. Endoscopic, radiologic and manometric correlation in small sliding hiatal hernia. Am J Gastroenterol. 1975;64:292-300.

3. Fornari F, Gurski RR, Navarini D, Thiesen V, Mestriner LH, Madalosso CA. Clinical utility of endoscopy and barium swallow $\mathrm{X}$-ray in the diagnosis of sliding hiatal hernia in morbidly obese patients: a study before and after gastric bypass. Obes Surg. 2010;20:702-8.

4. Bello B, Zoccali M, Gullo R, Allaix ME, Herbella FA, Gasparaitis A, et al. Gastroesophageal reflux disease and antireflux surgery-what is the proper preoperative work-up? J Gastrointest Surg. 2013;17:14-20.

5. Hill LD, Kozarek RA, Stefan JM, Aye RW, Mercer CD, Low DE, et al. The gastroesophageal flap valve: in vitro and in vivo observations. Gastrointest Endosc.
1996;44:541-7.

6. Jobe BA, Richter JE, Hoppo T, Peters JH, Bell R, Dengler WC, et al. Preoperative diagnostic work-up before antireflux surgery: an evidence and experience-based consensus of the Esophageal Advisory Panel. J Am Coll Surg. 2013;217:586-97.

7. Katz PO, Gerson LB, Vela MF. Guidelines for he diagnosis and management of gastroesophageal reflux disease. Am J Gastroenterol. 2013;108:308-28.

8. Pandolfino JE, Shi G, Trueworthy B, Kahrilas PJ. Esophagogastric junction opening during relaxation distinguishes non-hernia reflux patients, hernia patients and normals. Gastroenterology 2003;125:1018-24.

9. Kahrilas PJ, Kim HC, Pandolfino JE. Approaches to the Diagnosis and Grading of Hiatal Hernia. Best Pract Res Clin Gastroenterol. 2008;22:316-19.

10. Seltman AK, Kahrilas PJ, Chang EY, Mori M, Hunter JG, Jobe BA. Endoscopic measurement of cardia circumference as an indicator of GERD. Gastrointest Endosc. 2006;63:22-31

11. Pandolfino JE, Kim H, Ghosh S, Clarke JO, Zhang Q, Kahrilas PJ. High-Resolution
Manometry of the EGJ: An Analysis of Crural Diaphragm Function in GERD. Am J of Gastroent. 2007;102:1056-63.

12. Linke GR, Borovicka J, Schneider P, Zerz A, Warschkow R, Lange J, et al. Is a barium swallow complementary to endoscopy essential in the preoperative assessment of laparoscopic antireflux and hiatal hernia surgery? Surg Endosc. 2008;22:96-100.

13. Agrawal A, Tutuian R, Hila A, Freeman D, Castell DO. Identification of hiatal hernia by esophageal manometry. Is it reliable? Disease of the Esophagus 2005;18:316-9.

14. Klaus A, Raiser F, Swain JM, Hinder RA. Manometric components of the lower esophageal double hump. Dig Dis. 2000;18:172-7.

15. Weitzendorfer M, Köhler G, Antoniou SA, Pallwein-Prettner L, Manzenreiter L, Schredl P, et al. Preoperative diagnosis of hiatal hernia: barium swallow X-ray, highresolution manometry or endoscopy? Eur Surg. 2017;49:210-7.

16. Lindberg G. High resolution manometry change our views of gastrointestinal motility. Neurogastroenterol motil. 2013;25:780-2. 